\title{
Optimal power flow computations with a limited number of controls allowed to move
}

\author{
Florin Capitanescu, and Louis Wehenkel, Member, IEEE
}

\begin{abstract}
This letter focuses on optimal power flow (OPF) computations in which no more than a pre-specified number of controls are allowed to move. To determine an efficient subset of controls satisfying this constraint we rely on the solution of a mixed integer linear programming (MILP) problem fed with sensitivity information of controls' impact on the objective and constraints. We illustrate this approach on a 60-bus system and for the OPF problem of minimum cost to remove thermal congestion.
\end{abstract}

Index Terms-mixed integer linear programming, nonlinear programming, optimal power flow

\section{Motivation}

$\mathbf{S}$ YSTEM operators seek for a small number of control actions to take in a given time period to manage congestions or reach their operation goal in a given context [1]. Conventional OPF formulation uses the whole set of controls to solve the problem and very often most of them have moved at the optimal solution. The difficulty of limiting the number of controls moved in an OPF is due to the fact that: (i) almost every control variable participates in a non separable way to both improving the objective and satisfying the constraints, and (ii) the effectiveness of a control action is not necessarily related to its magnitude [1].

We propose an approach to limit the number of controls allowed to move in an OPF (called OPFLC in the sequel) based on MILP, first order sensitivities, and conventional OPF.

\section{OPFLC PROBLEM STATEMENT}

The OPFLC problem can be compactly stated as follows:

$$
\begin{array}{rl}
\min _{\mathbf{x}, \mathbf{u}, \mathbf{s}} & f(\mathbf{x}, \mathbf{u}) \\
\text { s.t. } & \mathbf{g}(\mathbf{x}, \mathbf{u})=\mathbf{0} \\
\mathbf{h}(\mathbf{x}, \mathbf{u}) \leq \mathbf{0} \\
s_{i}\left(\underline{u}_{i}-u_{i}^{0}\right) \leq u_{i}-u_{i}^{0} \leq s_{i}\left(\bar{u}_{i}-u_{i}^{0}\right) i=1, \ldots, n \\
\quad \sum_{i=1}^{n} s_{i} \leq N \\
\quad s_{i} \in\{0,1\} \quad i=1, \ldots, n,
\end{array}
$$

where $\mathbf{x}$ is the vector of state variables (i.e., real and imaginary part of bus voltages), $\mathbf{u}=\left[u_{1}, \ldots, u_{i}, \ldots, u_{n}\right]^{T}$ is the vector of control variables (e.g., generators active power, generators voltage, transformers ratio, shunts reactance, loads curtailment, phase shifters angle, etc.), vector $\underline{\mathbf{u}}$ (resp. $\overline{\mathbf{u}}$ ) denotes their

F. Capitanescu and L. Wehenkel are with the Department of Electrical Engineering and Computer Science, University of Liège, B4000 Liège, Belgium (e-mail: capitane@montefiore.ulg.ac.be; l.wehenkel@ulg.ac.be). lower (resp. upper) bounds and $\mathbf{u}^{0}$ their base case values, $\mathbf{s}$ is the vector of control variables statuses, $N$ is the maximum number of controls movements specified, $f(\cdot)$ is the objective function, and $\mathrm{g}(\cdot)$ and $\mathbf{h}(\cdot)$ model equality (e.g., the $\mathrm{AC}$ power flow equations) and inequality constraints (e.g., limits on branch currents and bus voltage magnitudes).

Constraints (4) bound control changes with respect to the base case. Note that if the status of a control is equal to 1 (resp. 0) the latter is allowed to vary between its bounds (resp. is frozen to its base case value $\left.u_{i}^{0}\right)$. Finally, constraints (5-6) limit the number of controls that can be changed.

The OPFLC problem (1-6) constitutes a mixed integer nonlinear programming problem (MINLP). To avoid the prohibitive computational time of classical MINLP approaches (e.g., branch and bound, Benders decomposition, etc.) and since the aim is to quickly obtain a near-optimal solution, faster heuristic techniques should be utilized.

\section{Proposed APPROACH TO THE OPFLC PROBLEM}

The key information of our approach is the sensitivity of the objective function and inequality constraints with respect to controls $\mathbf{u}$, which we denote by $\mathbf{S}_{\mathbf{u}}^{f}$ and $\mathbf{S}_{\mathbf{u}}^{\mathbf{h}}$. These sensitivities may be computed at the solution of a PF [7]:

$$
\begin{gathered}
\mathbf{S}_{\mathbf{u}}^{f}=\frac{\partial f}{\partial \mathbf{u}}-\left(\frac{\partial \mathbf{g}}{\partial \mathbf{u}}\right)^{T}\left[\left(\frac{\partial \mathbf{g}}{\partial \mathbf{x}}\right)^{T}\right]^{-1} \frac{\partial f}{\partial \mathbf{x}} \\
\mathbf{S}_{\mathbf{u}}^{\mathbf{h}}=\frac{\partial \mathbf{h}}{\partial \mathbf{u}}-\frac{\partial \mathbf{h}}{\partial \mathbf{x}}\left(\frac{\partial \mathbf{g}}{\partial \mathbf{x}}\right)^{-1} \frac{\partial \mathbf{g}}{\partial \mathbf{u}}
\end{gathered}
$$

The incremental computational effort needed to derive the sensitivities in (7) and (8) is very small, since the Jacobian $\partial \mathbf{g} / \partial \mathbf{x}$ is already available and factorized at the PF solution.

Then, the controls allowed to move are determined by solving the following sensitivity-based MILP problem:

$$
\begin{aligned}
& \min _{\mathbf{u}, \mathbf{s}}\left[f\left(\mathbf{x}^{0}, \mathbf{u}\right)-f\left(\mathbf{x}^{0}, \mathbf{u}^{0}\right)\right]=\min _{\mathbf{u}, \mathbf{s}} \sum_{i=1}^{n} S_{u_{i}}^{f}\left(u_{i}-u_{i}^{0}\right) \\
& \text { s.t. } h_{k}\left(\mathbf{x}^{0}, \mathbf{u}^{0}\right)+\sum_{i=1}^{n} S_{u_{i}}^{h_{k}}\left(u_{i}-u_{i}^{0}\right) \leq 0 \quad k=1, \ldots, d \\
& \quad s_{i}\left(\underline{u}_{i}-u_{i}^{0}\right) \leq u_{i}-u_{i}^{0} \leq s_{i}\left(\bar{u}_{i}-u_{i}^{0}\right) \quad i=1, \ldots, n \\
& \quad \sum_{i=1}^{n} s_{i} \leq N \\
& \quad s_{i} \in\{0,1\} \quad i=1, \ldots, n,
\end{aligned}
$$

where $d$ is the dimension of vector $\mathbf{h}$ in (3), and $S_{u_{i}}^{f}$ (resp. $S_{u_{i}}^{h_{k}}$ ) is a component of vector $\mathbf{S}_{\mathbf{u}}^{f}$ (resp. matrix $\mathbf{S}_{\mathbf{u}}^{\mathbf{h}}$ ). 
The objective (9) of the MILP minimizes the shift of function $f$ in (1) with respect to its initial value $f\left(\mathbf{x}^{0}, \mathbf{u}^{0}\right)$. Inequality constraints (10) linearize the original OPFLC constraints (3). The last three constraints are the same as (4-6).

Finally, the conventional OPF problem (1-4) is solved by using only those controls provided by the MILP problem.

For large systems and a large number of controls solving this MILP problem could be incompatible with real-time needs. In this case the MILP solver can be stopped earlier (e.g., as soon as the integrality gap becomes acceptable or when maximum running time is reached) yielding possibly a sub-optimal set of controls. Furthermore, to speed up computations, the linearized versions of constraints (2) have not been included into the MILP, assuming implicitly that these equations are satisfied also for the optimal values of controls provided by the MILP. Likewise, the MILP problem can be further simplified, e.g., by removing harmless constraints in (11) and inefficient control variables (i.e., with small sensitivities $S_{u_{i}}^{h_{k}}$ and/or a narrow range $\bar{u}_{i}-\underline{u}_{i}$ ).

\section{ILLUSTRATIVE EXAMPLE}

We illustrate the proposed approach on a 60-bus system [8]. We focus on the OPF problem of minimum load curtailment cost to remove thermal congestions. We assume the load curtailment is performed under constant power factor and does not exceed $10 \%$ of the total bus load.

We consider that following a line outage two branches are overloaded of $14 \%$ and $16 \%$, respectively. We also assume that these overloads have to be removed very quickly which prevents using slow controls such as generation rescheduling, and forces us to solve the problem by using the faster although more expensive load curtailment actions.

When solving the conventional OPF only 8 loads (out of all 22 loads) share the effort of overloads removal. Then we solve the MILP problem (9-13) for decreasing values of $N$, starting with $N=22$ and until the MILP becomes infeasible, situation which takes place for $N=1$. For each value of $N$, we solve the conventional OPF by allowing to move only the controls determined by the MILP. Figure 1 plots the OPF objective for various values of $N$. Clearly, the larger the number $N$ the better the objective. Note that the number of controls beyond which the objective can not be improved is $N=8$, i.e. the number of controls which has effectively moved in the conventional OPF. Obviously, for various values of $N$ different controls enter/leave the set of controls allowed to move.

The knowledge of the time horizon for reaching the operating goal allows the operator to estimate the maximum number of control actions $N$ that she/he could take, and hence to determine the best trade-off. The left part of the trade-off curve (obtained for $n<N$ ) allows her/him to assess the degree of sub-optimality implied by using smaller numbers of controls and whether there is enough room of manoeuvre in the case where some control actions would fail.

The CPU times (on a PC with 1.7-GHz and 512-Mb RAM) of various tasks of our approach are in average: $0.01 \mathrm{~s}$ for sensitivities computation, $0.03 \mathrm{~s}$ for MILP solution, and $0.12 \mathrm{~s}$ for the conventional OPF solution, respectively.

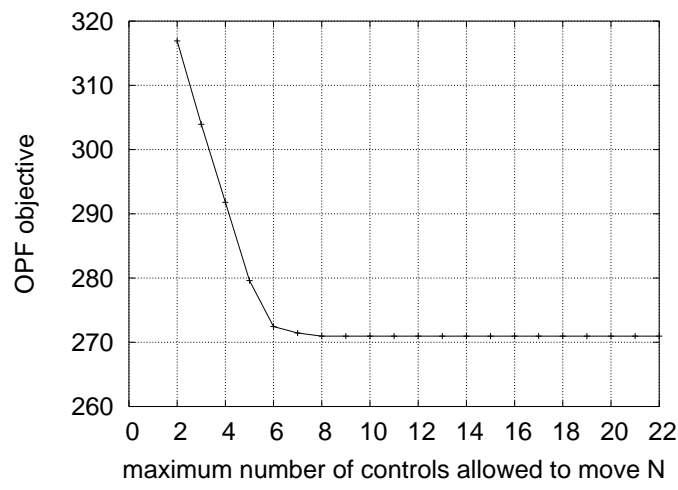

Fig. 1. Objective function versus number of controls allowed to move $N$

\section{RELATED WORK}

The few approaches proposed so far in the literature to the OPFLC problem rely on: beforehand specification of the controls participating in the OPF [1], sensitivity of the objective and constraints satisfaction to control movements [2], approximating the integral constraint of the maximal number of controls allowed to move by one nonlinear constraint [3], [4], mathematical programming with equilibrium constraints [4], embedding the DC approximation of the OPF into a MILP and focusing on topological actions [5], [6], respectively.

\section{CONCLUSION}

This letter has proposed a new formulation and solution approach to the OPF problem with a pre-specified number of controls allowed to move, relying on MILP and first order sensitivities. This tool is essential for the system operator, in the context of both operational planning and real-time, providing the desired number of controls so as to achieve a specified operation goal as well as a trade-off between the objective value and the number of control actions used.

Other experiments suggest that this approach behaves well also for reactive power dispatch (e.g., minimization of active power losses), although, due to slightly less accurate sensitivities values, the solutions are slightly more sub-optimal.

\section{REFERENCES}

[1] W.F. Tinney, J.M. Bright, K.D. Demaree, B.A. Hughes, "Some deficiencies in Optimal Power Flow", IEEE Trans. Power Syst., vol. 3, no. 2, 1988, pp. 676-683.

[2] S.A. Soman, K. Parthasarathy, and D. Thukaram, "Curtailed number and reduced controller movement optimization algorithms for real time voltage/reactive power control", IEEE Trans. Power Syst., vol. 9, no. 4, 1994, pp. 2035-2041.

[3] W.-H. Edwin Liu, and X. Gupa, "Fuzzy constraint enforcement and control action curtailement in an optimal power flow", IEEE Trans. Power Syst., vol. 11, no. 2, 1996, pp. 639-645.

[4] F. Capitanescu, W. Rosehart, and L. Wehenkel, "Optimal power flow computations with constraints limiting the number of control actions", Power Tech Conference, Bucharest (Romania), 2009.

[5] E.B. Fisher, R.P. O Neill, and M.C. Ferris, "Optimal transmission switching”, IEEE Trans. Power Syst., vol. 23, no. 3, 2008, pp. 1346-1355.

[6] S.A. Blumsack, "Network topologies and transmission investment under electric-industry restructuring", Ph.D. dissertation, Carnegie Mellon University, 2006.

[7] H.W. Dommel and W.F. Tinney, "Optimal power flow solutions", IEEE Trans. PAS, vol. PAS-87, no. 10, 1968, pp. 1866-1876.

[8] CIGRE Task Force 38.02.08, "Long-Term Dynamics, Phase II", 1995. 